\title{
Determination of the Gelificing Power of Maracuyá Shell Pectin Extracted in a Medium Acid and Its Application in Desserts
}

\section{Determinación del Poder Gelificante de Pectina Extraída de Cáscara de Maracuyá en Medio Ácido y Su Aplicación en Postres}

XVIII International Seminar on Health, Food and Human Nutrition

Corresponding Author:

Ana Matilde Moreno

amoreno@espoch.edu.ec

Published: 9 September 2021

Production and Hosting by

Knowledge E

(c) Leslie Catalina Rea Jara et al. This article is distributed under the terms of the Creative Commons Attribution License, which permits unrestricted use and redistribution provided that the original author and source are credited.

\section{Leslie Catalina Rea Jara ${ }^{1}$, Ana Matilde Moreno ${ }^{1}$, and Mayra Alexandra Logroño} Veloz ${ }^{2}$

${ }^{1}$ Escuela Superior Politécnica de Chimborazo, Facultad de Salud Pública, Carrera de Gastronomía, Riobamba, Ecuador

${ }^{2}$ Escuela Superior Politécnica de Chimborazo. Facultad de Salud Pública, Carrera de Nutrición y Dietética, Riobamba, Ecuador

\section{Abstract}

Introduction: Agro-industrial wastes contaminate on a large scale and some nutrients of functional importance found in the shells are not used, especially from fruits such as passion fruit (Passifloraedulisvar flavicarpa). Objective: The gelling power of pectin extracted with acid hydrolysis was determined by two treatments from passion fruit peel and pulp and its application in desserts. Methodology: The extraction was carried out from the shell: T1 with hydrochloric acid and T2 with citric acid. Subsequently, the gelling power was measured comparing with commercial pectin and the extracted pectin was applied to make two mousse desserts $(0 \%$, $25 \%$ and $50 \%)$ and jam (0\%, 0.5\%, $1 \%$ pectin) evaluated with acceptability tests applying a hedonic scale test. Results: T2 presents a higher percentage of pectin extraction yield 15.14 \pm 1.22 with a $(p>0.05)$ with respect to T1; likewise, T2 shows greater gelling power with better consistency of the gel formed with $21 \mathrm{~g}$ of sugar at $65^{\circ}$ Brix compared to commercial pectin with $38^{\circ}$ Brix. The jam prepared with $1 \%$ extracted pectin showed a score of 7 : I like it moderately with a semi-soft, spreadable texture, a light color, flavor and characteristic aroma of the fruit. The mousse with 50\% extracted pectin scored 8: I like it moderately. Conclusion: Pectin is a polysaccharide of industrial and gastronomic interest, it was possible to extract it under controlled conditions of $\mathrm{pH}$, time and temperature by hydrolysis with citric acid with better gelling power than commercial pectin.

Keywords: acid hydrolysis, pectin, gelling agent.

\section{Resumen}

Introducción: Los desechos agroindustriales contaminan a gran escala y no se aprovechan algunos nutrientes de importancia funcional que se encuentran en las cáscaras, especialmente de frutas como el maracuyá (Passifloraedulisvar flavicarpa). Objetivo: Se determinó el poder gelificante de pectina extraída con hidrólisis ácida por dos tratamientos a partir de cáscara y pulpa del maracuyá y aplicación en postres. Metodología: La extracción se realizó de la cáscara: T1 con ácido clorhídrico y T2 con ácido cítrico. Posteriormente se midió el poder de gelificación comparando con pectina comercial y se aplicó la pectina extraída para elaborar 
dos postres mousse $(0 \%, 25 \%$ y $50 \%$ ) y mermelada $(0 \%, 0,5 \%, 1 \%$ de pectina) evaluados con pruebas de aceptabilidad aplicando un test de escala hedónica. Resultados: EI T2 presenta mayor porcentaje de rendimiento de extracción de pectina 15,14 $\pm 1,22$ con un $(p>0,05)$ con respecto a T1; asimismo, T2 muestra mayor poder gelificante con mejor consistencia del gel formado con $21 \mathrm{~g}$ de azúcar a $65^{\circ}$ Brix comparando con pectina comercial con $38^{\circ}$ Brix. La mermelada preparada con $1 \%$ de pectina extraída mostró una puntuación de 7: Me gusta moderadamente con textura semiblanda, untable, color tenue sabor y aroma característico de la fruta. El mousse con $50 \%$ de pectina extraída obtuvo puntuación de 8 : Me gusta moderadamente. Conclusiones: La pectina es un polisacárido de interés industrial y gastronómico se logró extraer en condiciones controladas de $\mathrm{pH}$, tiempo y temperatura por hidrólisis con ácido cítrico con mejor poder gelificante que la pectina comercial.

Palabras Clave: hidrólisis ácida, pectina, gelificante.

\section{Introducción}

La pectina es un polisacárido tecnológicamente funcional [1], por sus características reológicas que aportan consistencia y textura apropiada en las preparaciones gastronómicas y procesos industriales como en la elaboración de jaleas (fabricadas con pectina de bajo grado de esterificación) mermeladas, gelatinas (con pectinas de alta esterificación) salsas entre otros [2].

Químicamente la estructura básica de la pectina está integrada por moléculas de al menos el $65 \%$ de ácido D-galacturònico unidas por enlaces glucosídicos $\alpha-D[1,4]$, [3] en el cual algunos de los carboxilos pueden estar esterificados con metilos o en formas de sal (Figura 1); además, se puede encontrar asociado a otros carbohidratos especialmente con hemicelulosa, en las paredes de los vegetales responsables de la firmeza de algunos productos y la disolución de los componentes de la pared celular especialmente de la pectina se ha relacionado con el ablandamiento de varios alimentos [4].

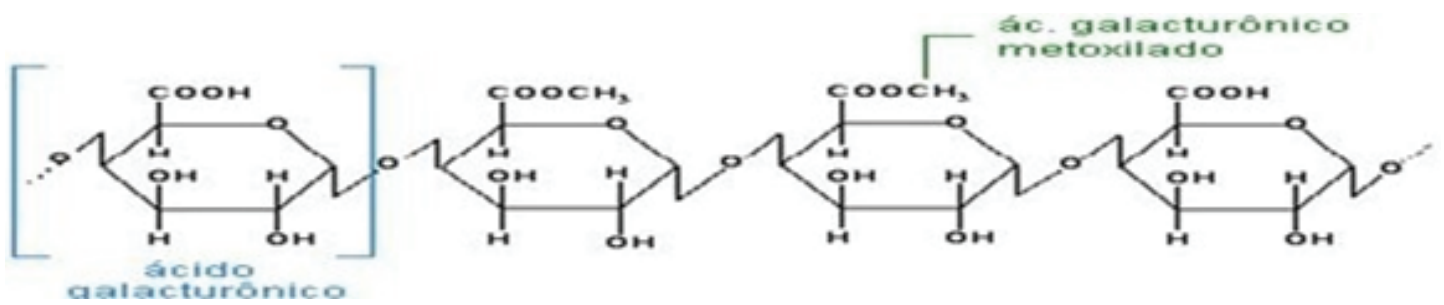

Figure 1

Estructura química de la pectina. Fuente: Pectina - EcuRed 2020 Enciclopedia Cubana [5].

Existen algunas características importantes que definen la calidad de las pectinas y son: (1) Grado de metoxilación y (2) oder gelificante; [6] el primero refiere a la esterificación de grupos carboxilos por radicales metilos, de este modo se dividen en pectinas de alto metoxilo (comprende más del 50\% con ácido galacturónico) de las cuales constan de rápida y lenta gelificación y las de bajo metoxilo (menos del 50\% están 
esterificadas con metanol) las cuales requieren la presencia de cationes divalentes como el calcio [4].

Mientras que el poder gelificante es la capacidad que tiene la pectina para formar geles [7] por ejemplo una pectina de grado 150 significa que $1 \mathrm{~kg}$ de pectina podrá gelificar 150 kg de azúcar [8].

Aunque el mecanismo exacto de la formación del gel no está claro, se han hecho progresos significativos en esta dirección. Dependiendo de la pectina, coordinar la unión con $\mathrm{Ca} 2+$ Los iones o enlaces de hidrógeno y las interacciones hidrofóbicas están implicados en la formación del gel [8].

La transcendental aplicación de la pectina es la elaboración de mermeladas, compotas, gelificante en pudines, estabilizante de emulsiones, agente viscosante en bebidas y estabilizante de helados y postres fríos [9].

La pectina es una sustancia que se puede encontrar en todas las frutas y vegetales; y puede hallarse de forma simple en pequeñas cantidades y en forma de gel en mayores dosis, la cual tiene una función depurativa considerada como una fibra soluble. Las frutas que presentan mayor concentración de pectina son: Membrillos, cítricos, manzanas y maracuyá [8]. Se puede considerar como aditivo alimentario [9].

Existen otras interesantes fuentes alternativas para la extracción de pectina a partir de residuos industriales que son provenientes del procesamiento de papaya, mango, melocotón, girasol, café y cacao [10].

Los subproductos generados, la cáscara y la pulpa, cuando se depositan en los suelos se consideran un foco para la propagación de un hongo del género Phytophora spp, el cual es el causante principal de pérdidas económicas de la actividad de industrias de alimentos [11].

El maracuyá presenta un $50-60 \%$ de peso en la cáscara, jugo de 30-40\% y las semillas $10-15 \%$, de lo cual sólo se consume el zumo y la cáscara no se aprovecha convirtiéndose en un desecho agroindustrial de importancia.

El objetivo de la presente investigación fue determinar el poder gelificante de la pectina extraída por hidrólisis ácida por dos tratamientos con ácido clorhídrico y ácido cítrico a partir de la cáscara y su aplicación en la elaboración de postres.

\section{Metodología}

El tipo de investigación fue experimental y descriptiva, desarrollada en la carrera de Gastronomía de la Facultad de Salud pública de la ESPOCH. El objeto de estudio fue el maracuyá de variedad amarilla (Passifloraedulisvar flavicarpa) con un índice de madurez que exhibió las siguientes características: Cáscara color amarillo dura, sólidos solubles $13^{\circ}$ Brix mínimo, color de la pulpa amarilla - rojiza y sabor - aromas característicos sin indicios de fermentación.

El proceso de extracción se efectuó a partir de la cáscara con dos tratamientos; T1 extracción con ácido clorhídrico y T2 extracción con ácido cítrico. Posterior se midió el poder de gelificación comparando con pectina comercial y se aplicó la pectina extraída en la elaboración de dos postres mousse $(0,25$ y 50\%) y mermelada( $0,0,5$, 
$1 \%$ de pectina) evaluados con pruebas de aceptabilidad por un test de escala hedónica aplicados a consumidores potenciales [8].

El tratamiento estadístico se realizó con el software de versión libre 2017 InfoStat.

\subsection{Proceso de extracción de la pectina}

Se presenta en forma secuencial el diagrama para la extracción de la pectina por hidrólisis ácida a partir de la cáscara del maracuyá.
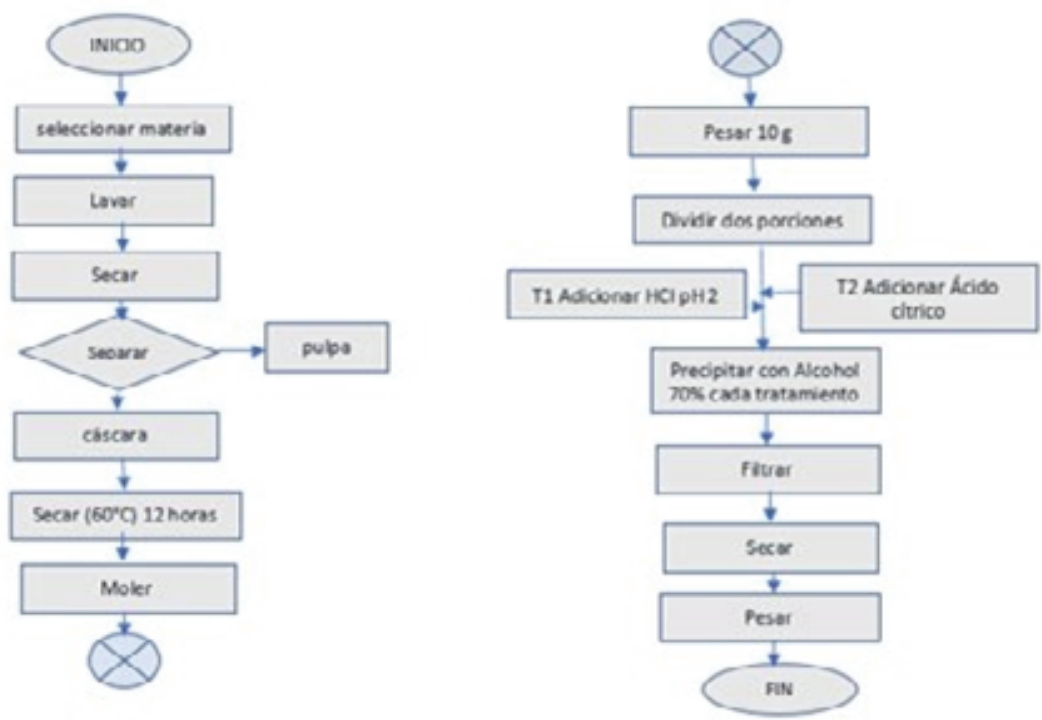

Figure 2

T1: Extracción de pectina por hidrólisis ácida. Fuente: Rea Jara LC (2014) [8].

\subsection{Proceso para determinar el poder gelificante}

Para medir el poder gelificante ${ }^{\circ} \mathrm{S}$ la pectina extraída del maracuyá se gelificó conjuntamente con la sacarosa y ácido cítrico hasta alcanzar un pH de 3,2, y se comparó con la pectina comercial, seguidamente se midió los ${ }^{\circ}$ Brix. Se aplicó la siguiente fórmula:

$$
{ }^{\circ} \mathrm{S}=\frac{\text { Masa de sólidos }(\text { sacarosa })}{\text { Masa de pectina }}=65^{\circ} \text { Brix }
$$

\subsection{Condiciones de los postres usando la pectina extraída del maracuyá}

\subsubsection{Elaboración de mermelada}

Para la cocción de la mermelada se consideró la aplicación de Buenas prácticas de manufactura de acuerdo a las normas ARCSA [12]. 


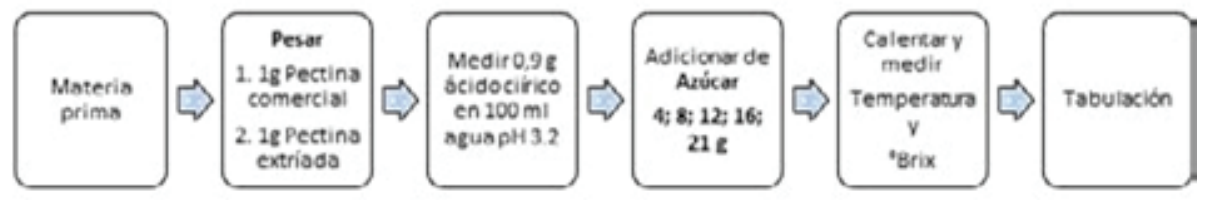

Figure 3

Determinación del poder gelificante. Fuente: Rea Jara LC (2014) [8].

Formulación: 45\% pulpa, 55\% de azúcar, 0,05\% de ácido cítrico; variando: 0, 0,5 y $1 \%$ de pectina.

Acidez: $\mathrm{pH}$ 3,3-3,75.

Punto de gelificación: 65-68 Brix.

Se midió el tiempo y la temperatura de la cocción variando según la cantidad y el uso de pectina comercial y pectina extraída y aplicó un test de escala hedónica para medir la aceptabilidad [8].

\subsubsection{Elaboración de mousse}

En la Tabla 1 se indica la formulación para $50 \mathrm{~g}$ de mousse con tres tratamientos: 0, 25, $50 \%$ se aplicó un test de escala hedónica para evaluar la aceptabilidad.

\section{Table 1}

Formulación de mousse.

\begin{tabular}{l|l|l|l|l|l|} 
Tratamientos & Gelificante & $\begin{array}{l}\text { Pectina } \\
\text { extraída }\end{array}$ & $\begin{array}{l}\text { Pulpa } \\
\text { maracuyá }\end{array}$ & $\begin{array}{l}\text { Leche } \\
\text { condensada }\end{array}$ & $\begin{array}{l}\text { Leche } \\
\text { evaporada }\end{array}$ \\
\hline T1 & $0 \%$ & $0 \%$ & $20 \%$ & $40 \%$ & $40 \%$ \\
T2 & $75 \%$ & $25 \%$ & $15 \%$ & $30 \%$ & $30 \%$ \\
T3 & $50 \%$ & $50 \%$ & $15 \%$ & $30 \%$ & $30 \%$ \\
\hline
\end{tabular}

Fuente: Rea Jara LC (2014) [8].

\section{Resultados}

\subsection{Porcentaje de rendimiento de extracción de la pectina por hidrólisis ácida}

Como se indica en la Tabla 2, el análisis de varianza muestra que existen diferencias significativas comparando los dos tratamientos, siendo el T2 la extracción con ácido cítrico el que obtuvo mayor porcentaje de rendimiento (Figura 5) para el proceso de extracción de la pectina, por ser un ácido grado alimenticio, en cambio el $\mathrm{HCl}$ no se puede usar en concentraciones elevadas, lo que implica baja cantidad de pectina de extracción. 
Table 2

Analisis de varianza del porcentaje de rendimiento de extracción de la pectina por dos tratamientos: T1: (HCl) y T2: (ácido cítrico).

\begin{tabular}{|c|c|c|c|c|c|}
\hline \multirow{2}{*}{\multicolumn{6}{|c|}{$\begin{array}{lllll}\text { Variable } & N R^{2} & R^{2} & \mathrm{Al} \\
\text { \% RFNDIMIFNTO DF PFCTINA F.. } & 10 & 0.94 & 0.87\end{array}$}} \\
\hline & & & & & \\
\hline \multicolumn{6}{|c|}{ Cuadro de Análisis de la Varianza (SC tipo I) } \\
\hline F.V. & $\mathrm{SC}$ & gl & $\mathrm{CM}$ & $\mathrm{F}$ & p-valor \\
\hline Modelo & 488.83 & 5 & 97.77 & 13.21 & 0.0135 \\
\hline TRATAMIENTOS & 429.94 & 1 & 429.94 & 58.10 & 0.0016 \\
\hline REPLICAS & 58.89 & 4 & 14.72 & 1.99 & 0.2608 \\
\hline Frror & 29.60 & 4 & 7.40 & & \\
\hline Total & 518.44 & $\underline{9}$ & & & \\
\hline \multicolumn{6}{|c|}{ Test: Duncan Alfa-0.05 } \\
\hline TRATAMIENTOS & Medias & n & E.E. & & \\
\hline T1 & 2.03 & 5 & 1.22 & A & \\
\hline$\underline{\mathrm{T} 2}$ & 15.14 & 5 & 1.22 & B & \\
\hline
\end{tabular}

Medias con una letra común no son significativamente diferentes $(p>0,05)$.

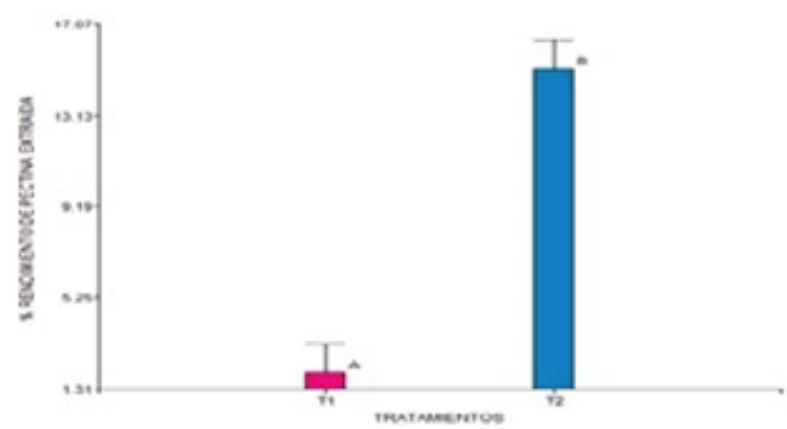

Figure 4

Porcentaje de rendimiento de extracción de pectina.

\subsection{Poder gelificante}

Se observa en la Tabla 3 y la Figura 5 existe una correlación entre la cantidad de sacarosa y los sólidos solubles sin importar la cantidad de pectina; pero si depende del tipo de pectina, así se aprecia que con la pectina extraída se necesita menor cantidad de sacarosa para la gelificación de $21 \mathrm{~g}$ de azúcar alcanza $65^{\circ}$ Brix pero para la pectina comercial se necesitaron $25 \mathrm{~g}$ para legar a los $65^{\circ} \mathrm{Brix}$, consistencias de solidos solubles adecuada para una buena textura de gel.

El azúcar tiende a deshidratar las moléculas de pectina en solución y cuanta más cantidad de este soluto, hay menos cantidad de agua disponible para solubilizar la pectina, por lo tanto, se favorece la tendencia a gelificar [13]. 
Table 3

Poder gelificante de pectina comercial y pectina extraída $\mathrm{pH}$ 3,2.

\begin{tabular}{|c|c|c|c|}
\hline Pectina (g) & Sacarosa (g) & Pectina comercial ${ }^{\circ}$ Brix & Pectina extraída ${ }^{\circ}$ Brix \\
\hline 1 & 4 & 5,5 & 5 \\
\hline 1 & 8 & 9,5 & 10 \\
\hline 1 & 12 & 15 & 16 \\
\hline 1 & 16 & 21 & 23 \\
\hline 1 & 17 & 26,5 & 32 \\
\hline 1 & 21 & 36,8 & 65,2 \\
\hline 1 & 25 & 65 & 68 \\
\hline
\end{tabular}

Fuente: Rea Jara LC (2014) [8].

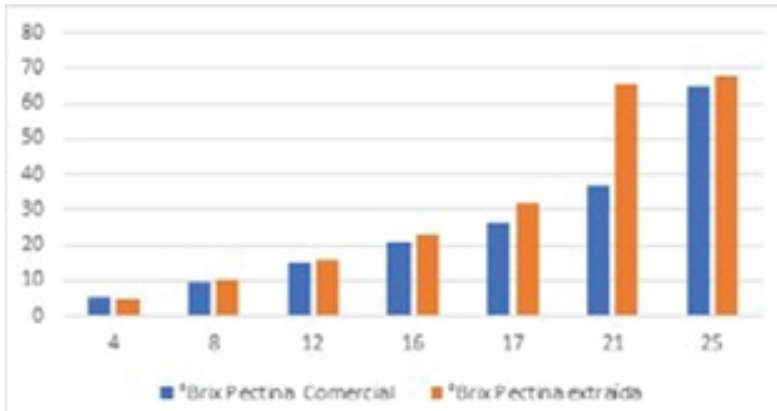

Figure 5

Comparación de ${ }^{\circ}$ Brix para medir el poder de gelificación. Fuente: Rea Jara LC (2014) [8].

\subsection{Aceptabilidad de los postres con el uso de la pectina extraída}

La pectina extraída incide en las características organolépticas de la mermelada, es responsable de reaccionar con el azúcar actúa como espesante durante la cocción y al momento de enfriarse se solidifica, lo que incide en cuanto a la apariencia [14]. La muestra T3 con 1\% de pectina exteriorizó una textura semiblanda, untable agradable al gusto con un color tenue, no incide en el aroma ni en el color porque depende de la fruta base (Figura 5). En promedio los consumidores prefieren la mermelada en la mayoría de los atributos con una puntuación que se encuentra en me gusta moderadamente con una puntuación promedio de 7 .

Una característica de un mousse es el uso de agares o gelatinas para dar el aspecto gelificado, no es común usar pectina, pero en este caso la adición de la pectina extraída reemplazando en un 50\%, incurre en las características organolépticas; exhibiendo una textura cremosa y esponjosa con apariencia homogénea, color y aromo característico del maracuyá, con un puntaje de aceptabilidad de la textura de 8 puntos me gusta modernamente en una escala hedónica de rango de 1 a 9 (Figura 6). 


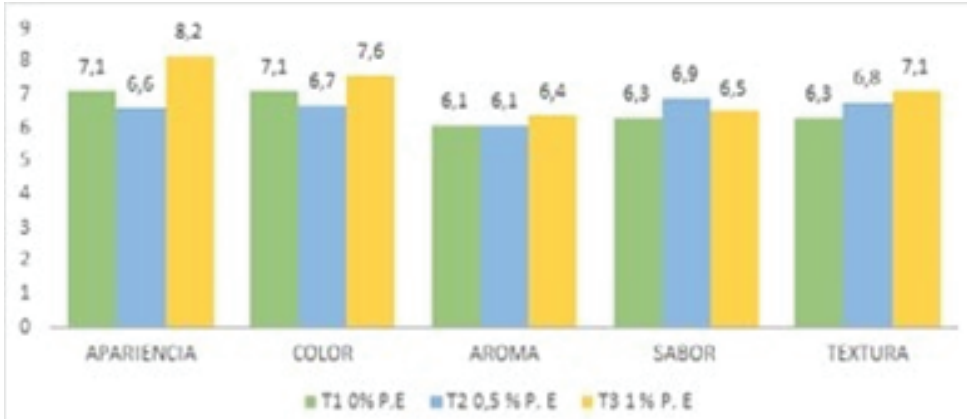

Figure 6

Aceptabilidad de mermelada con pectina extraída de la cáscara del maracuyá. Fuente: Rea Jara LC (2014) [8].

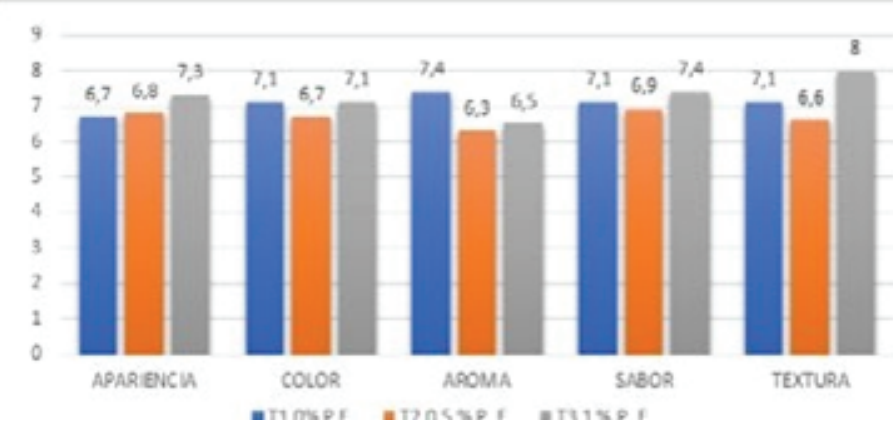

Figure 7

Aceptabilidad de mousse con pectina extraída de la cáscara del maracuyá. Fuente: Rea Jara LC (2014) [8].

\section{Discusión}

La demanda por parte de consumidores de productos saludables y la necesidad de minimizar la contaminación al ambiente, incentiva a los investigadores a explorar las formas de usar subproductos que genera la industria agroalimentaria y de esta manera obtener ingredientes funcionales, como es caso de las pectinas que es un polisacárido que tiene propiedades biológicas que lo hace útil no solo en la industria alimentaria sino también en la farmacéutica por las propiedades prebióticas y tecno funcionales debido a su poder gelificante y estabilizante [15].

El dar valor agregado a los desechos orgánicos que no se aprovecha es una alternativa útil para generar un producto más natural y su aplicación en la gastronomía.

Otro aspecto de importancia es que las pectinas son termorreversibles en presencia de sacarosa a pH bajos, por su óptima capacidad de gelificación es responsable de la textura de los productos vegetales y viscosidad. Además, se usa como sustituto de grasas y azúcares en productos bajos en calorías [16].

En Honduras existe una empresa que procesa 400.000 frutos de maracuyá anualmente lo que genera $21.200 \mathrm{~kg}$ de cáscara, que en su mayoría se usa como abono orgánico; y para optimizar el uso de la materia prima investigaron con un diseño 
completamente al azar (DCA) con dos agentes de extracción similares a los realizados en esta investigación (ácido clorhídrico y ácido cítrico), con cuatro tratamientos y 12 unidades experimentales, el rendimiento promedio de extracción con ácido cítrico fue de $21,25 \%$, comparando con el resultado obtenido en este proceso solo se obtuvo con un promedio de $16 \%$, ya que se debe tomar en cuenta la temperatura y el tiempo de hidrólisis, lo realizan por 120 min y en este experimento solo se hidrolizó con un tiempo máximo de 30 min. Otro aspecto de comparación es que la pectina extraída sólo presento propiedad significativa en cuanto a la viscosidad, pero no a su poder de gelificación contrario a la pectina que se extrajo en menor tiempo en este experimento [17].

La pectina tiene un $\mathrm{pH}$ de 3,5 valor considerado como tope, y de debe tomar en cuenta ya que si el pH naja se obtienen geles de pectina de alto metoxilo y por lo tanto un mejor rendimiento de la materia prima [13]

En otra investigación se extrajo pectina mediante hidrólisis ácida de las moras de castilla dando un poder gelificante medio-alto con un rendimiento de $14 \%$ y es una pectina que contribuye a mejorar el aspecto y la textura de los postres con un porcentaje de puntuación elevado de aceptabilidad por parte de los consumidores [18].

Racón-Chu et al., en el trabajo de investigación 'Gelificación iónica de pectina de bajo grado de esterificación extraída de manzanas inmaduras de raleo', extrajeron pectina mediante hidrólisis ácida controlada por valores de $\mathrm{pH}$, con rendimientos de $12 \%$ en peso y manifiestan que se puede usar en industria alimentaria. [19] de forma similar en nuestra investigación, al controlar la acidez aumenta el porcentaje de rendimiento.

Barazarte y col, utilizaron cáscaras de cacao para producción de pectina a nivel comercial con diferentes condiciones de $\mathrm{pH}$ y temperatura y como medio de precipitación el EDTA; obtuvieron un rendimiento de extracción de 2,64 a 4,7 g/100 g. Rendimiento muy bajo comparado con nuestra investigación, lo que indica que el uso de ácido cítrico mejora condiciones de extracción, además prepararon una mermelada con un nivel de agrado promedio de 'me gusta moderadamente', se recomienda optimizar los parámetros de extracción para aumentar el rendimiento [20].

Zegada V. efectuó dos métodos de extracción de pectina a partir de cáscaras de naranja, por el método convencional y por hidrólisis ácida asistida por microondas, lo que mejoró en tiempo y se podría aplicar a nivel industrial. Aplicando este método se pretende generar un ahorro de energía y tiempo en el proceso de producción de pectina [21].

Por otro lado, también se puede extraer la pectina de forma líquida a partir de cáscaras de frutos cítricos proporcionando como resultado una elevada capacidad de gelificación siempre y cuando se controle la temperatura, tiempo de hidrólisis, $\mathrm{PH}$ facilitando rendimientos óptimos con características reológicas de interés para la industria y la gastronomía [22]. 


\section{Conclusiones}

La pectina es un polisacárido de interés industrial y gastronómico que se puede extraer en condiciones controladas de $\mathrm{PH}$ tiempo y temperatura generalmente por hidrólisis ácida para menorar el PH y la capacidad de gelificación a partir de desechos agroindustriales como las cáscaras del maracuyá.

\section{References}

[1] Addosio DR, Ferrer G, Páez M, Marín JZM. Obtención y caracterización de pectina a partir de la cáscara de parchita (Passiflora edulis f. flavicarpa Degener). Rev Facutad Agronómica. 2005;22(3):241-51.

[2] Seixas, F. Extraction of pectin from passion fruit peel (Passiflora edulis f. flavicarpa) by microwaveinduced heating. Food Hydrocoll. 2014;38:186-92.

[3] Badui S, Badui D. Química de los alimentos [Internet]. $4^{\text {th }}$ ed. Wesley PA, editor. Mèxico: Pearson Education; 2006 [cited 2020 Mar 13]. Available from: www.pearsoneducacion.net

[4] Guidi A, Arandia-Quiroga M. Obtención de pectina a partir de la cáscara de maracuyá por hidrólisis ácida. J Boliv Ciencias Fac Informática y Electrónica-Tecnología-Arquitectura y Tur. 2010;7(21):68-70.

[5] EcuRed Enciclopedia Cubana. Pectina [Internet]. 2020 [cited 2020 Mar 13]. Available from: https://www. ecured.cu/EcuRed:Enciclopedia_cubana

[6] Urango-Anaya KJ, Ortega-Quintana FA, Vélez-Hernández G, Pérez-Sierra ÓA. Extracción Rápida de Pectina a Partir de Cáscara de Maracuyá (Passiflora edulis flavicarpa) empleando Microondas. Inf Tecnológica. 2018;29(1):129-36.

[7] Liu, L. Extraction of pectins with different degrees of esterification from mulberry branch bark. Bioresour Technol. 2010;100(9):3268-73.

[8] Rea JLC. Determinación del poder gelificante de la pectina extraída de la cáscara y pulpa del maracuyá (Passiflora edulis f. flavicarpa I.) para elaboración de postres [Internet]. Escuela Superior Politécnica de Chimborazo. 2014. Available from: http://dspace.espoch.edu.ec/handle/123456789/9814

[9] Norma Técnica Ecuatoriana NTE INEN-CODEX STAN. Norma General del Codex para los aditivos alimentarios [Internet]. 1995 [cited 2020 Aug 4]. Available from: http://www.who.int/ipcs/food/jecfa/en/

[10] Voragen A. Advances in Pectin and Pectinase research. Springer; 2003.

[11] Franco-Castillo M, Ramírez-Hernández M, García-Gómez RS, Bernal-González M, Espinosa-Aquino B. Reaprovechamiento integral de resíduos agroindustriales; cáscaras y pulpa de cacao para la producción de pectinas. 2010;1(2):45-66.

[12] Ministerio de Salud Pública. Reglamento de Etiquetado de Alimentos Procesados para el Consumo Humano. Acuerdo No 00004522 [Internet]. 2013;0(00004522):8. https://www.controlsanitario.gob.ec/ wp-content/uploads/downloads/2014/08/

[13] Saravia, PC, Nass RD, Suazo EV. Pectina: química, fuentes, proceso de extracción, gelificación y su uso en la elaboración de mermeladas o jaleas y otras aplicaciones. 2014.

[14] Instituto Ecuatoriano de Normalización (INEN) NTE INEN 2825. Norma para las confituras, jaleas y mermeladas (CODEX STAN 296-2009, MOD). 2009.

[15] Muñoz A. Caracterización de pectinas industriales de cítricos y su aplicación como recubrimientos de fresas (Tesis de Master en Quimica Agricola Y Nuevos Alimentos). 2016;1-75.

[16] Puigvert JY, Garza S. Los geles de pectina y su aplicación en la industria alimentaria. Aliment Rev Tecnol e Hig los Aliment. 2003;232:93-8.

[17] Ochoa, LRC, Cojulun R. Efecto del agente de extracción y tiempo de hidrólisis ácida en el rendimiento de pectina de cáscaras de maracuyá (Passiflora edulis var. flavicarpa). Esc AGRÍcOLA Panam Honduras [Internet]. 2008; http://www.sidalc.net/cgi-bin/wxis.exe/?IsisScript=zamocat.xis\&method= post\&formato $=2 \&$ cantidad $=1 \&$ expresion $=\mathrm{mfn}=024432$

[18] Valencia MAP. Extracción y aplicación de pectina de mora en la elaboración de recetas reposteras tipo gourmet. Escuela Superior Politécnica de Chimborazo; 2012.

[19] Toribio MR, De-Amazonj S, Maldonado B, et al. Extracción de pectina mediante el método de hidrólisis ácida en frutos de maushan (vasconcellea weberbaueri (harms)v.m. Badillo) en dos índices de madurez provenientes del distrito de San Miguel de Soloco, región Amazonas. [Internet]. Universidad Nacional Toribio Rodríguez de Mendoza de Amazonas; 2010 Available from: http://repositorio.untrm.edu.pe/ handle/UNTRM/959

[20] Barazarte H, Sangronis E, Unai E. La cáscara de cacao (Theobroma cacao L.): Una posible fuente comercial de pectinas [Internet]. Vol. 58. Organo Oficial de la Sociedad Latinoamericana de Nutrición; 2008. Available from: https://pdfs.semanticscholar.org/09bc/d5c71e4ce841b88dd04dfbfbc3e75c08fd8d.pdf 
[21] Zegada FV. Extracción de pectina de residuos de cáscara de naranja por hidrólisis ácida asistida por microondas (HMO). Investig Desarro. 2015;1(15).

[22] Alexandra M, Ávila R. Extracción de pectina líquida a partir de cáscaras de Maracuyá (Passiflora edulis) y su aplicación en el desarrollo de un producto de humedad intermedia. Guayaquil; 2009. Available from: https://www.dspace.espol.edu.ec/bitstream/123456789/11411/4/ ExtracciondePectinaLiquidaapartirdelacascaradeMaracuya\{\%\}28Passifloraedulis\{\%\}29ysuaplicac2.pdf 